\title{
ON A NEW KIND OF EINSTEIN WARPED PRODUCT (POLJ)-MANIFOLD
}

\author{
A. PIGAZZINI, C. ÖZEL ${ }^{\dagger}$, P. LINKER AND S. JAFARI
}

\author{
Date of Receiving : $\quad$ 02.05.2019 \\ Date of Revision : $\quad 10.08 .2019$ \\ Date of Acceptance : 03.09 .2019
}

\begin{abstract}
We provide a possible way of constructing a new kind of Einstein warped-product manifold which we will call POLJ-manifold; if we can build the fiber-manifold as a derived-differential-manifold (i.e. the fiber-manifold can admit negative dimension), then we have an Einstein warped product manifold that may have zero dimension $(\operatorname{dim} B+\operatorname{dim} F=0)$ so the result may be an "invisible"manifold but made up of two manifolds with 2 and -2 dimension respectively, a kind of "point-manifold" (zero dimension) with "hidden" dimensions. The POLJmanifold could introduce a new kind of Kaluza-Klein theory with extra negative dimensional spaces, or Kaluza-Klein theory with new kind of time-manifold. In fact POLJ-manifold could describe a new nature of time.
\end{abstract}

\section{Introduction and Preliminaries}

The concept of negative dimensional space is already used in linguistic statistics [2]. Also in supersymmetric theories in Quantum Field Theory, negative dimensional spaces were used [3].

Let $E \cong M \times F$ be a fiber bundle with base space $M$ and its fiber $F$. We will discuss now a case, where the fiber has negative dimension. Note that the total dimension of the fiber bundle is given by the relation $\operatorname{dim} E=\operatorname{dim} M+\operatorname{dim} F$. We will consider the case, where the base manifold has greater positive dimension than the negative dimension of the fiber, i.e. $\operatorname{dim} M>-\operatorname{dim} F$. In this case, the dimension of the total fiber bundle is still positive. Since the base manifold is obtained by projection of the fiber bundle along the fiber by projection operator $\pi_{F}$, we have $\pi_{F} E=M$ i.e. the projection of the lower-dimensional fiber-bundle along the fiber yields the higher dimensional base manifold space. Therefore, the projection operator $\pi_{F}$ along the negative-dimensional fiber, is a suspension operator that raises the dimension of topological spaces.

2010 Mathematics Subject Classification. 53C25.

Key words and phrases. Einstein warped product, derived-differentials, negative dimensional manifolds, invisible-manifolds, point-manifolds, derived-manifold.

Communicated by: S. P. Moshokoa

${ }^{\dagger}$ Corresponding author. 